PROCEEDINGS OF THE

AMERICAN MATHEMATICAL SOCIETY

Volume 139, Number 4, April 2011, Pages 1163-1174

S 0002-9939(2010)10781-5

Article electronically published on December 6, 2010

\title{
MOTIVIC INVARIANTS OF ALGEBRAIC TORI
}

\author{
JOHANNES NICAISE \\ (Communicated by Ted Chinburg)
}

\begin{abstract}
We prove a trace formula and a global form of Denef and Loeser's motivic monodromy conjecture for tamely ramified algebraic tori over a discretely valued field. If the torus has purely additive reduction, the trace formula gives a cohomological interpretation for the number of components of the Néron model.
\end{abstract}

\section{INTRODUCTION}

Let $R$ be a complete discrete valuation ring, with quotient field $K$ and residue field $k$. We assume that $k$ is algebraically closed. We denote by $p$ the characteristic exponent of $k$, and by $\mathfrak{M}$ the maximal ideal of $R$. We fix a separable closure $K^{s}$ of $K$, and we denote by $K^{t}$ the tame closure of $K$ in $K^{s}$. We denote by $I$ the inertia group $G\left(K^{s} / K\right)$, by $P \subset I$ the wild inertia subgroup, and by $I^{t}=I / P=G\left(K^{t} / K\right)$ the tame inertia group. We fix a prime $\ell \neq p$ and a topological generator $\sigma$ of $I^{t}$.

We denote by $K_{0}\left(\operatorname{Var}_{k}\right)$ the Grothendieck ring of $k$-varieties, by $\mathbb{L}=\left[\mathbb{A}_{k}^{1}\right]$ the class of the affine line in $K_{0}\left(\operatorname{Var}_{k}\right)$, and by $\mathcal{M}_{k}$ the localization of $K_{0}\left(\operatorname{Var}_{k}\right)$ w.r.t. $\mathbb{L}$. We denote by

$$
\chi_{\text {top }}: K_{0}\left(\operatorname{Var}_{k}\right) /(\mathbb{L}-1) \rightarrow \mathbb{Z}
$$

the $\ell$-adic Euler characteristic (it is independent of $\ell$ ). See [11, 2.1] for details.

If $R$ has equal characteristic, then we put $K_{0}^{R}\left(\operatorname{Var}_{k}\right)=K_{0}\left(\operatorname{Var}_{k}\right)$ and $\mathcal{M}_{k}^{R}=$ $\mathcal{M}_{k}$. If $R$ has mixed characteristic, we denote by $K_{0}^{R}\left(\operatorname{Var}_{k}\right)$ the modified Grothendieck ring of $k$-varieties [15, 3.2]. It is a quotient of $K_{0}\left(\operatorname{Var}_{k}\right)$, obtained by identifying the classes of universally homeomorphic $k$-varieties. With a slight abuse of notation, we denote the image of $\mathbb{L}$ in $K_{0}^{R}\left(\operatorname{Var}_{k}\right)$ again by $\mathbb{L}$; we will always clearly indicate in which ring we are working. We denote by $\mathcal{M}_{k}^{R}$ the localization of $K_{0}^{R}\left(\operatorname{Var}_{k}\right)$ with respect to $\mathbb{L}$. The Euler characteristic $\chi_{\text {top }}$ factors through $K_{0}^{R}\left(\operatorname{Var}_{k}\right) /(\mathbb{L}-1)$.

Let $X$ be a separated rigid $K$-variety. A weak Néron model for $X$ is a separated smooth formal $R$-scheme $\mathfrak{U}$, topologically of finite type, endowed with an open immersion of rigid $K$-varieties $i: \mathfrak{U}_{\eta} \rightarrow X$ such that $i(K): \mathfrak{U}_{\eta}(K) \rightarrow X(K)$ is a bijection. Here we denote by $\mathfrak{U}_{\eta}$ the generic fiber of $\mathfrak{U}$. Such a weak Néron model exists iff $X$ admits a smooth quasi-compact open rigid subvariety which contains

Received by the editors April 14, 2009 and, in revised form, April 8, 2010.

2000 Mathematics Subject Classification. Primary 14G10, 20G25, 14F20.

Key words and phrases. Motivic Serre invariant, trace formula, motivic zeta function, monodromy conjecture.

The author was partially supported by ANR-06-BLAN-0183.

(C)2010 American Mathematical Society Reverts to public domain 28 years from publication 
all the $K$-points of $X$ [3, 3.3]. A weak Néron model is far from being unique in general. Nevertheless, using motivic integration on formal schemes, it was shown in [7, 4.5.3] and [14, 5.11] (see also [15, §5.3] for an erratum) that the class

$$
\left[\mathfrak{U}_{s}\right] \in K_{0}^{R}\left(\operatorname{Var}_{k}\right) /(\mathbb{L}-1)
$$

of the special fiber $\mathfrak{U}_{s}$ of $\mathfrak{U}$ only depends on $X$, and not on $\mathfrak{U}$. It is called the motivic Serre invariant of $X$ and is denoted by $S(X)$. We consider $S(X)$ as a measure for the set of rational points on $X$. In particular, $S(X)$ vanishes if $X(K)=\emptyset$ (but the converse implication does not hold). If $Y$ is an algebraic $K$-variety such that $Y^{a n}$ admits a weak Néron model, we put $S(Y)=S\left(Y^{a n}\right)$ (here $(\cdot)^{a n}$ is the non-Archimedean GAGA functor). This definition applies in particular when $Y$ is smooth and proper.

We showed in [11, 6.3] that the motivic Serre invariant admits a cohomological interpretation by means of a trace formula: if $Y$ is a smooth and proper $K$-variety, then under a certain tameness condition on $Y$ (in particular if $k$ has characteristic zero), we have

$$
\chi_{\text {top }}(S(Y))=\sum_{i \geq 0}(-1)^{i} \operatorname{Trace}\left(\sigma \mid H^{i}\left(Y \times_{K} K^{t}, \mathbb{Q}_{\ell}\right)\right) .
$$

An analogous statement holds for rigid varieties [10,6.4]. It seems plausible that (1.1) holds for any smooth, proper, geometrically connected algebraic $K$-variety $Y$ such that the wild inertia $P$ acts trivially on the $\ell$-adic cohomology of $Y$ and such that $Y\left(K^{t}\right)$ is non-empty. The results in [11, $\S 7$ ] show that this is true when $Y$ is a curve, and that the assumption $Y\left(K^{t}\right) \neq \emptyset$ cannot be dropped. We proved in [12] the case where $Y$ is an abelian variety.

In this paper we consider the case of a tamely ramified algebraic $K$-torus $G$. Its analytification $G^{a n}$ does not admit a weak Néron model in general, so the motivic Serre invariant has to be defined in another way. There are two possible definitions. First, $G^{a n}$ has a natural quasi-compact open rigid subgroup $G^{b}$ such that $G^{b}(K)$ is the maximal subgroup of $G(K)$ which is bounded in $G$ [6, 3.12]. We show in Theorem 3.8 that (1.1) holds for $G$ if the left hand side is replaced by $\chi_{\text {top }}\left(S\left(G^{b}\right)\right.$ ). Second, if $K$ has characteristic zero, we showed in [11, 5.4] (see also [15, $§ 5.3$ ] for an erratum) that there exists a unique ring morphism

$$
S: K_{0}\left(\operatorname{Var}_{K}\right) \rightarrow K_{0}^{R}\left(\operatorname{Var}_{k}\right) /(\mathbb{L}-1)
$$

such that $S([Y])=S(Y)$ for any smooth and proper $K$-variety $Y$. If $X$ is a $K$ variety such that $X^{a n}$ admits a weak Néron model, we have $S([X])=S\left(X^{a n}\right)$ by 11. 5.4]. We show in Proposition 3.3 that, if $K$ has characteristic zero and $G$ is an algebraic $K$-torus, we have $S([G])=S\left(G^{b}\right)$. In fact, the analogous equality holds for any algebraic $K$-group $H$ for which $H^{b}$ can be defined.

In [6], we associated a motivic zeta function $Z_{A}(T)$ to every semi-abelian $K$ variety $A$. It measures the behaviour of the Néron model of $A$ under tame base change. If $G$ is a tamely ramified algebraic $K$-torus, then the trace formula gives a cohomological interpretation of $Z_{G}(T)$ (Proposition 4.3). We prove in Theorem6.1 that $G$ satisfies a global version of Denef and Loeser's monodromy conjecture for motivic zeta functions of complex hypersurface singularities. More precisely, we show that $Z_{G}\left(\mathbb{L}^{-s}\right)$ has a unique pole at $s=c(G)$, where $c(G)$ denotes the base change conductor of $G$ [4. This pole has order one, and $\exp (2 \pi i c(G))$ belongs to $\{-1,1\}$ and is the unique eigenvalue of $\sigma$ on $H^{g}\left(G \times_{K} K^{t}, \mathbb{Q}_{\ell}\right)$, with $g$ the 
dimension of $G$. We also compute the characteristic polynomial of the $\sigma$-action on $H^{1}\left(G \times_{K} K^{t}, \mathbb{Q}_{\ell}\right)$ in terms of the elementary divisors $c_{i}(G)$ of $G$ (Proposition 3.5). We refer to [6] for analogous results for abelian varieties and for the exact relation with Denef and Loeser's motivic monodromy conjecture.

The assumption that $G$ is tamely ramified is crucial for the arguments in this paper. It would be highly interesting to adapt the results to the wildly ramified case.

\section{Preliminaries}

We denote by $\mathbb{N}^{\prime}$ the set of integers $d>0$ prime to $p$. For each $d \in \mathbb{N}^{\prime}$, we denote by $K(d)$ the unique degree $d$ extension of $K$ in $K^{t}$, by $I(d)$ the subgroup $G\left(K^{s} / K(d)\right)$ of $I$, and by $I^{t}(d)$ the subgroup $G\left(K^{t} / K(d)\right)$ of $I^{t}$.

Let $G$ be an algebraic torus over $K$. We denote by $g$ the dimension of $G$ and by $X$ the character group of $G$. It is a free $\mathbb{Z}$-module of rank $g$, endowed with a continuous action of the inertia group $I=G\left(K^{s} / K\right)$. The splitting degree of $G$ is the degree of the minimal extension $L$ of $K$ where $G$ splits. We say that $G$ is tamely ramified if the wild inertia $P$ acts trivially on $X$. This is equivalent to the property that the splitting degree of $G$ is prime to $p$, i.e. that $G$ splits over a finite tame extension of $K$.

For any element $\gamma$ of $I^{t}$, we denote by $P_{\gamma}(t)$ the characteristic polynomial

$$
\operatorname{det}\left(t \cdot I d-\gamma \mid H^{1}\left(G \times_{K} K^{t}, \mathbb{Q}_{\ell}\right)\right)
$$

of the action of $\gamma$ on $H^{1}\left(G \times_{K} K^{t}, \mathbb{Q}_{\ell}\right)$. Since there exists an $I^{t}$-equivariant isomorphism

$$
H^{1}\left(G \times_{K} K^{t}, \mathbb{Q}_{\ell}\right) \cong\left(X \otimes_{\mathbb{Z}} \mathbb{Q}_{\ell}\right)^{P},
$$

the polynomial $P_{\gamma}(t)$ coincides with the characteristic polynomial of the $\gamma$-action on $X^{P} \otimes_{\mathbb{Z}} \mathbb{Q}$. In particular, $P_{\gamma}(t)$ belongs to $\mathbb{Z}[t]$, and it is a product of cyclotomic polynomials, independent of $\ell$.

An algebraic $K$-group is a group $K$-scheme of finite type. When we speak of the Néron model of an algebraic $K$-group $H$, we mean the Néron model defined in [6, 3.6]. By [6, 3.10], a smooth commutative algebraic $K$-group $H$ admits a Néron model $\mathcal{H}$ iff it admits a Néron $l f t$-model $\mathcal{H}^{l f t}$ in the sense of [2, 10.1.1]. This condition is also equivalent to the property that $H$ does not admit a subgroup of type $\mathbb{G}_{a, K}[2,10.2 .2]$. The Néron model $\mathcal{H}$ is the maximal quasi-compact open subgroup $R$-scheme of $\mathcal{H}^{l f t}$ [6, 3.7]. We denote by $\Phi_{H}$ the group of connected components of the special fiber $\mathcal{H}_{s}$ and by $\Phi_{H}^{\prime}$ the subgroup of elements of order prime to $p$. The group $\Phi_{H}$ is the torsion part of the component group of $\mathcal{H}_{s}^{l f t}$. We denote by $H^{b}$ the generic fiber of the formal $\mathfrak{M}$-adic completion of $\mathcal{H}$. Then $H^{b}$ is a separated smooth quasi-compact rigid $K$-variety, and $H^{b}(K)$ is the maximal subgroup of $H(K)$ which is bounded in $H$ [6, 3.12].

We denote by $\mathcal{G}$ the Néron model of the $K$-torus $G$. The identity component $\mathcal{G}_{s}^{o}$ of $\mathcal{G}_{s}$ splits canonically into a product $T \times_{k} U$ with $T$ a $k$-torus and $U$ a unipotent $k$-group. The dimensions of $T$ and $U$ are called the toric, resp. unipotent, rank of $\mathcal{G}_{s}^{o}$. We say that $G$ has good reduction if $U$ is trivial and that $G$ has purely additive reduction if $T$ is trivial.

For each integer $d>0$, we denote by $\Phi_{d}(t) \in \mathbb{Z}[t]$ the cyclotomic polynomial whose zeroes are the primitive $d$-th roots of unity. For every rational number $a$, we denote by $\tau(a)$ the order of $a$ in the quotient group $\mathbb{Q} / \mathbb{Z}$. 


\section{The motivic Serre invariant And the trace formula}

Lemma 3.1. Let $H$ be a smooth connected commutative algebraic $k$-group and consider its Chevalley decomposition

$$
0 \longrightarrow\left(L=U \times_{k} T\right) \longrightarrow H \stackrel{\pi}{\longrightarrow} B \longrightarrow 0
$$

with $U$ unipotent, $T$ a torus, and $B$ an abelian variety. If we denote by $u$ and $t$ the dimensions of $U$, resp. $T$, then

$$
[H]=\mathbb{L}^{u}(\mathbb{L}-1)^{t}[B]
$$

in $K_{0}\left(\operatorname{Var}_{k}\right)$.

Proof. As a $k$-variety, $U$ is isomorphic to $\mathbb{A}_{k}^{u}$ [18, VII, $\left.\mathrm{n}^{\circ} 6\right]$. By the scissor relations in the Grothendieck ring, it suffices to show that $\pi$ is a Zariski-locally trivial fibration. But $\pi$ is an $L$-torsor with respect to the $f p p f$ topology, and hence also with respect to the Zariski topology, because $L$ is a successive extension of $\mathbb{G}_{m}$ and $\mathbb{G}_{a}$ [8, III.3.7 and III.4.9].

Lemma 3.2. Let $H$ be a smooth commutative algebraic $K$-group. Assume that $H$ admits a Néron model $\mathcal{H}$, and denote by $t$ the dimension of the maximal torus in $\mathcal{H}_{s}^{o}$. If $H$ admits a subgroup $T \cong \mathbb{G}_{m, K}$, then $t>0$.

Proof. Denote by $\mathcal{T}$ the Néron model of $T$. If $t=0$, then any morphism of group $k$-schemes $\mathbb{G}_{m, k} \rightarrow \mathcal{H}_{s}^{o}$ is trivial. Since $\mathcal{T}_{s}^{o} \cong \mathbb{G}_{m, k}$, it suffices to show that the natural morphism $f: \mathcal{T}_{s}^{o} \rightarrow \mathcal{H}_{s}^{o}$ is non-trivial. For each integer $q$ prime to $p$, we have a commutative diagram

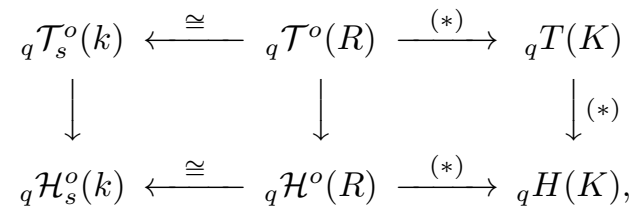

where the lower index ${ }_{q}(\cdot)$ denotes the kernel of multiplication by $q$. The left horizontal morphisms are bijections by [2, 7.3.3], and all arrows marked by (*) are injective. It follows that the map

$$
{ }_{q} \mathcal{T}_{s}^{o}(k) \longrightarrow{ }_{q} \mathcal{H}_{s}^{o}(k)
$$

is injective. But $\mathcal{T}_{s}^{o}$ is isomorphic to $\mathbb{G}_{m, k}$, so that ${ }_{q} \mathcal{T}_{s}^{o}(k)$ is non-trivial. Hence, $f$ is non-trivial.

Proposition 3.3. Let $H$ be a smooth commutative algebraic $K$-group and assume that $H$ admits a Néron model $\mathcal{H}$. Denote by $t$ the dimension of the maximal torus in $\mathcal{H}_{s}^{o}$ and by $B$ the abelian quotient in the Chevalley decomposition of $\mathcal{H}_{s}^{o}$. Then

$$
S\left(H^{b}\right)=\left\{\begin{array}{cc}
\left|\Phi_{H}\right| \cdot[B] & \text { if } t=0, \\
0 & \text { otherwise. }
\end{array}\right.
$$

Moreover, if $K$ has characteristic zero, then $S([H])=S\left(H^{b}\right)$.

Proof. We start by computing $S\left(H^{b}\right)$. The $\mathfrak{M}$-adic completion of $\mathcal{H}$ is a weak Néron model for $H^{b}$, so that $S\left(H^{b}\right)=\left[\mathcal{H}_{s}\right]$ in $K_{0}^{R}\left(\operatorname{Var}_{k}\right) /(\mathbb{L}-1)$. Since $k$ is algebraically closed, every connected component of $\mathcal{H}_{s}$ is isomorphic to $\mathcal{H}_{s}^{o}$. By Lemma 3.1. [ $\mathcal{H}_{s}$ ] vanishes in $K_{0}^{R}\left(\operatorname{Var}_{k}\right) /(\mathbb{L}-1)$ unless $t=0$, and in the latter case, $\left[\mathcal{H}_{s}\right]=\left|\Phi_{H}\right| \cdot[B]$ 
in $K_{0}^{R}\left(\operatorname{Var}_{k}\right) /(\mathbb{L}-1)$. Hence, it suffices to show that $S([H])=S\left(H^{b}\right)$, assuming that $K$ has characteristic zero.

Assume that $H$ admits a subgroup $T \cong \mathbb{G}_{m, K}$. Consider the short exact sequence of $K$-groups

$$
1 \longrightarrow T \longrightarrow H \stackrel{f}{\longrightarrow} H / T \longrightarrow 1 \text {. }
$$

Since $T$ is a split torus, $f$ is a $T$-torsor in the Zariski topology, and in particular a Zariski-locally trivial fibration with fiber $T$. This implies that $[H]=[T][H / T]$ in $K_{0}\left(\operatorname{Var}_{K}\right)$. Since the motivic Serre invariant

$$
S(\cdot): K_{0}\left(\operatorname{Var}_{K}\right) \rightarrow K_{0}^{R}\left(\operatorname{Var}_{k}\right) /(\mathbb{L}-1)
$$

maps $\left[\mathbb{G}_{m, K}\right]$ to zero $[11,5.4]$, we see that $S([H])=0$. On the other hand, $t>0$ by Lemma 3.2, so that $S\left(H^{b}\right)=0$ as well.

Now, assume that $H$ does not admit a subgroup of type $\mathbb{G}_{m, K}$. Then the Néron lft-model $\mathcal{H}^{l f t}$ is quasi-compact [2, 10.2.1], so that $\mathcal{H}=\mathcal{H}^{l f t}$, and $H^{b}(K)=H(K)$. Hence, the $\mathfrak{M}$-adic completion of $\mathcal{H}$ is a weak Néron model of $H^{\text {an }}$, and we have

$$
S([H])=S\left(H^{b}\right)=\left[\mathcal{H}_{s}\right] \quad \in K_{0}^{R}\left(\operatorname{Var}_{k}\right) /(\mathbb{L}-1)
$$

by [11, 5.4].

Now we turn to our algebraic $K$-torus $G$.

Lemma 3.4. Let $L$ be the minimal splitting field of $G$. The component group $\Phi_{G}$ is killed by the splitting degree $e=[L: K]$ of $G$, and $\left|\Phi_{G}\right|=\left|H^{1}(G(L / K), X)\right|$.

Proof. The component group $\Phi_{G}$ is isomorphic to $H^{1}(I, X)$, by [1, 7.2.2] and [19, 2.18]. The $I$-action on $X$ factors through $G(L / K)$. Since $X$ is torsion-free, the inflation morphism

$$
H^{1}(G(L / K), X) \rightarrow H^{1}(I, X)
$$

is an isomorphism [17, VII, Prop. 4]. The group $H^{1}(G(L / K), X)$ is killed by $e$, by 17. VIII, Cor. 1].

Proposition 3.5. The following are equivalent:

(1) $G$ has purely additive reduction.

(2) $P_{\sigma}(1) \neq 0$.

(3) $P_{\sigma}(1)=\left|\Phi_{G}^{\prime}\right|$.

Proof. The implication $(3) \Rightarrow(2)$ is trivial. It follows from [9, 1.3] that the torus $G$ has purely additive reduction iff $X^{I}=0$. This proves the equivalence of (1) and (2). It remains to show that $(1) \Rightarrow(3)$. Assume that $G$ has purely additive reduction, denote by $L$ the minimal splitting field of $G$, and by $L^{\prime}$ the maximal tame extension of $K$ inside $L$. By [17, VII, Prop. 4], we have an exact sequence

$$
0 \rightarrow H^{1}\left(G\left(L^{\prime} / K\right), X^{P}\right) \rightarrow H^{1}(G(L / K), X) \rightarrow H^{1}\left(G\left(L / L^{\prime}\right), X\right) .
$$

We have $\left|H^{1}(G(L / K), X)\right|=\left|\Phi_{G}\right|$ by Lemma 3.4. Since $H^{1}\left(G\left(L / L^{\prime}\right), X\right)$ is a $p$ group, and $H^{1}\left(G\left(L^{\prime} / K\right), X^{P}\right)$ has order prime to $p$ [17, VIII, Cor. 1], we find that

$$
\left|\Phi_{G}^{\prime}\right|=\left|H^{1}\left(G\left(L^{\prime} / K\right), X^{P}\right)\right|=\left|X^{P} /(1-\sigma) X^{P}\right|,
$$

where the last equality follows from [17, VIII, Prop. 6] and the fact that $X^{I}=0$.

By [17, III, Prop. 2] we have

$$
\left|X^{P} /(1-\sigma) X^{P}\right|=\left|\operatorname{det}\left(1-\sigma \mid X^{P} \otimes_{\mathbb{Z}} \mathbb{Q}\right)\right| .
$$


The polynomial $P_{\sigma}(t)$ equals the characteristic polynomial of $\sigma$ on $X^{P} \otimes_{\mathbb{Z}} \mathbb{Q}$. It is a product of cyclotomic polynomials that do not vanish at $t=1$, which easily implies that $P_{\sigma}(1)>0$ (see [12, 2.6]). Hence, we find that

$$
\left|\Phi_{G}^{\prime}\right|=P_{\sigma}(1) \text {. }
$$

Corollary 3.6. If $G$ has purely additive reduction, then $\left|\Phi_{G}^{\prime}\right|$ is invariant under isogeny.

Corollary [3.6] is false without the assumption that $G$ has purely additive reduction, as is shown by the following easy example.

Example 3.7. Assume that $k$ has characteristic zero. Let $G_{1}$ and $G_{2}$ be the $K$-tori whose character group is $\mathbb{Z}^{2}$ with $\sigma$-action given by the matrices

$$
\left(\begin{array}{ll}
0 & 1 \\
1 & 0
\end{array}\right) \text {, resp. }\left(\begin{array}{cc}
1 & 0 \\
0 & -1
\end{array}\right) \text {. }
$$

Then $G_{1}$ and $G_{2}$ are isogenous, but computing $H^{1}(I, X)$ for both tori we find that $\Phi_{G_{1}}=\{0\}$ while $\Phi_{G_{2}}=\mathbb{Z} / 2 \mathbb{Z}$.

Theorem 3.8 (Trace formula). Assume that $G$ is tamely ramified. Then $\Phi_{G}^{\prime}=\Phi_{G}$. Moreover, if $G$ has purely additive reduction, then

$$
\chi_{\text {top }}\left(S\left(G^{b}\right)\right)=\left|\Phi_{G}\right|=\sum_{i \geq 0}(-1)^{i} \operatorname{Trace}\left(\sigma \mid H^{i}\left(G \times_{K} K^{t}, \mathbb{Q}_{\ell}\right)\right) .
$$

Otherwise,

$$
\chi_{\text {top }}\left(S\left(G^{b}\right)\right)=0=\sum_{i \geq 0}(-1)^{i} \operatorname{Trace}\left(\sigma \mid H^{i}\left(G \times_{K} K^{t}, \mathbb{Q}_{\ell}\right)\right) .
$$

Proof. Since $G$ is tamely ramified, the splitting degree $e$ of $G$ is prime to $p$, so $\Phi_{G}^{\prime}=\Phi_{G}$ by Lemma 3.4. By Proposition 3.3 we find that

$$
S\left(G^{b}\right)=\left|\Phi_{G}\right| \quad \in K_{0}^{R}\left(\operatorname{Var}_{k}\right) /(\mathbb{L}-1)
$$

if $G$ has purely additive reduction, and $S\left(G^{b}\right)=0$ otherwise.

By tameness of $G$, we have

$$
H^{i}\left(G \times_{K} K^{t}, \mathbb{Q}_{\ell}\right)=H^{i}\left(G \times{ }_{K} K^{s}, \mathbb{Q}_{\ell}\right)^{P}=H^{i}\left(G \times_{K} K^{s}, \mathbb{Q}_{\ell}\right)
$$

for each $i \geq 0$, so there exists a canonical $I^{t}$-equivariant isomorphism

$$
H^{i}\left(G \times_{K} K^{t}, \mathbb{Q}_{\ell}\right) \cong \bigwedge_{\mathbb{Q}_{\ell}}^{i} H^{1}\left(G \times_{K} K^{t}, \mathbb{Q}_{\ell}\right)
$$

for each $i \geq 0$. It follows easily that

$$
\sum_{i \geq 0}(-1)^{i} \operatorname{Trace}\left(\sigma \mid H^{i}\left(G \times_{K} K^{t}, \mathbb{Q}_{\ell}\right)\right)=P_{\sigma}(1),
$$

and we may conclude the proof by Proposition 3.5 .

Corollary 3.9. If $K$ has characteristic zero and $G$ is tamely ramified, then

$$
\chi_{\text {top }}(S([G]))=\sum_{i \geq 0}(-1)^{i} \operatorname{Trace}\left(\sigma \mid H^{i}\left(G \times_{K} K^{t}, \mathbb{Q}_{\ell}\right)\right) .
$$

For abelian varieties, we proved an analogue of Theorem 3.8 in [12, 2.5 and 2.8]. 


\section{The MOtivic Zeta FUnCtion of A tORUS}

For any field $F$ and any smooth $F$-scheme $Z$ of pure dimension, a gauge form on $Z$ is a nowhere vanishing differential form of maximal degree. If $Y$ is a smooth $R$-scheme of pure relative dimension, $\phi$ a gauge form on $Y \times_{R} K$ and $C$ a connected component of the special fiber $Y_{s}$, then we denote by $\operatorname{ord}_{C} \omega$ the order of $\phi$ along $C$ [6, 2.1].

For any $d \in \mathbb{N}^{\prime}$ we denote by $\mathcal{G}(d)$ the Néron model of $G(d)=G \times_{K} K(d)$. We denote by $\omega$ a distinguished gauge form on $G$ [6, 7.1], i.e. a translation-invariant gauge form such that $\operatorname{ord}_{\mathcal{G}_{s}^{\circ} \omega}=0$. Such a distinguished gauge form always exists, and it is unique up to multiplication with a unit in $R[2,4.2 .3]$. For any $d \in \mathbb{N}^{\prime}$ we denote by $\omega(d)$ the pull-back of $\omega$ to $G(d)$. We introduce a function

$$
\operatorname{ord}_{G}: \mathbb{N}^{\prime} \rightarrow \mathbb{N}
$$

by putting $\operatorname{ord}_{G}(d)=-\operatorname{ord}_{\mathcal{G}(d)_{s}^{o}} \omega(d)$. This function only depends on $G$ and not on $\omega$. The fact that ord $_{G}$ takes its values in the positive integers follows from [6, 7.4].

The following definition is taken from [6, §8.1].

Definition 4.1. We define the motivic zeta function $Z_{G}(T)$ of $G$ by

$$
Z_{G}(T)=\sum_{d \in \mathbb{N}^{\prime}}\left[\mathcal{G}(d)_{s}\right] \mathbb{L}^{\operatorname{ord}_{G}(d)} T^{d} \in \mathcal{M}_{k}[[T]] .
$$

For each $d \in \mathbb{N}^{\prime}$, the image of $\left[\mathcal{G}(d)_{s}\right] \mathbb{L}^{\operatorname{ord}_{G}(d)}$ in $\mathcal{M}_{k}^{R}$ can be interpreted in terms of a motivic integral of the "Haar measure" $\omega(d)$ on the bounded part $G(d)^{b}$ of $G(d)$ : we have

$$
\left[\mathcal{G}(d)_{s}\right] \mathbb{L}^{\operatorname{ord}_{G}(d)}=\mathbb{L}^{g} \cdot \int_{G(d)^{b}}|\omega(d)| \in \mathcal{M}_{k}^{R}
$$

In order to give a more explicit description of $Z_{G}(T)$, we introduce some notation. For each $d \in \mathbb{N}^{\prime}$, we put $\phi_{G}(d)=\left|\Phi_{G(d)}\right|$, the number of connected components of $\mathcal{G}(d)_{s}$. Moreover, we denote by $t_{G}(d)$ and $u_{G}(d)$ the toric, resp. unipotent, rank of $\mathcal{G}(d)_{s}^{o}$. Of course, we have $t_{G}(d)+u_{G}(d)=g$ for every $d \in \mathbb{N}^{\prime}$.

\section{Proposition 4.2.}

$$
Z_{G}(T)=\sum_{d \in \mathbb{N}^{\prime}} \phi_{G}(d)(\mathbb{L}-1)^{t_{G}(d)} \mathbb{L}^{u_{A}(d)+\operatorname{ord}_{G}(d)} T^{d} \in \mathcal{M}_{k}[[T]] .
$$

Proof. This is immediate from Lemma 3.1

The trace formula yields the following cohomological interpretation of $Z_{G}(T)$. We denote by $\chi_{t o p}\left(Z_{G}(T)\right)$ the series in $\mathbb{Z}[[T]]$ obtained by applying the morphism $\chi_{\text {top }}: \mathcal{M}_{k} \rightarrow \mathbb{Z}$ to the coefficients of $Z_{G}(T)$.

Proposition 4.3. If $G$ is tamely ramified, then

$$
\begin{aligned}
\chi_{\text {top }}\left(Z_{G}(T)\right) & =\sum_{d \in \mathbb{N}^{\prime}, t_{G}(d)=0} \phi_{G}(d) T^{d} \\
& =\sum_{d \in \mathbb{N}^{\prime}} \sum_{i \geq 0}(-1)^{i} \operatorname{Trace}\left(\sigma^{d} \mid H^{i}\left(G \times_{K} K^{t}, \mathbb{Q}_{\ell}\right)\right) T^{d} .
\end{aligned}
$$

Proof. This is immediate from Theorem 3.8 and Proposition 4.2 
By Proposition 4.2, the motivic zeta function $Z_{G}(T)$ only depends on the functions $\phi_{G}, t_{G}, u_{G}$ and $\operatorname{ord}_{G}$ from $\mathbb{N}^{\prime}$ to $\mathbb{N}$. We will now recall how the first three of them can be computed from the $I$-module $X$.

Proposition 4.4. For each $d \in \mathbb{N}^{\prime}$ we have

$$
\begin{aligned}
\phi_{G}(d) & =\left|H^{1}(I(d), X)\right|, \\
t_{G}(d) & =\operatorname{rank}_{\mathbb{Z}}\left(X^{I(d)}\right), \\
u_{G}(d) & =\operatorname{rank}_{\mathbb{Z}}\left(X / X^{I(d)}\right) .
\end{aligned}
$$

Proof. It obviously suffices to prove the result for $d=1$, since $G(d)$ is the torus corresponding to the character group $X$ with the action of $I(d)$. The first equality follows from [1, 7.2.2] and [19, 2.12]; the other two follow easily from [9, 1.3].

Corollary 4.5. Assume that $G$ is tamely ramified, and denote by e the splitting degree of $G$. Then $\phi_{G}(d), u_{G}(d)$ and $t_{G}(d)$ only depend on $d$ mod e. More precisely, if $d$ is an element of $\mathbb{N}^{\prime}$ and $e^{\prime}$ is a multiple of e such that $d+e^{\prime} \in \mathbb{N}^{\prime}$, then

$$
\begin{aligned}
\phi_{G}\left(d+e^{\prime}\right) & =\phi_{G}(d), \\
u_{G}\left(d+e^{\prime}\right) & =u_{G}(d), \\
t_{G}\left(d+e^{\prime}\right) & =t_{G}(d) .
\end{aligned}
$$

Proof. Since $G$ is tamely ramified, we may replace $I(d)$ by $I^{t}(d)$ in Proposition 4.4 , Note that $I^{t}(d)$ is topologically generated by $\sigma^{d}$, and $I^{t}\left(d+e^{\prime}\right)$ by $\sigma^{d+e^{\prime}}$. But $\sigma^{e}$ acts trivially on $X$, so the actions of $\sigma^{d}$ and $\sigma^{d+e^{\prime}}$ on $X$ coincide.

We recall the following definition [4, 2.4].

Definition 4.6. Let $L$ be the minimal splitting field of $G$. Denote by $R^{\prime}$ the normalization of $R$ in $L$, and by $\mathfrak{M}^{\prime}$ the maximal ideal of $R^{\prime}$. Put $e=[L: K]$ and $G^{\prime}=G \times_{K} L$, and denote by $\mathcal{G}^{\prime}$ the Néron model of $G^{\prime}$. By the universal property of the Néron model, there exists a unique morphism of $R^{\prime}$-schemes

$$
\mathcal{G} \times{ }_{R} R^{\prime} \rightarrow \mathcal{G}^{\prime}
$$

that extends the isomorphism between the generic fibers. We have an isomorphism of $R^{\prime}$-modules

$$
\operatorname{Lie}\left(\mathcal{G}^{\prime}\right) / \operatorname{Lie}\left(\mathcal{G} \times{ }_{R} R^{\prime}\right) \cong \bigoplus_{i=1}^{v}\left(R^{\prime} /\left(\mathfrak{M}^{\prime}\right)^{c_{i} \cdot e}\right)
$$

with $c_{1} \leq \ldots \leq c_{v}$ in $(1 / e) \mathbb{Z}_{>0}$. The tuple $\left(c_{1}, \ldots, c_{v}\right)$ is called the tuple of elementary divisors of $G$, and

$$
c(G):=\sum_{i=1}^{v} c_{i}=\frac{1}{e} \cdot \operatorname{length}_{R^{\prime}}\left(\operatorname{Lie}\left(\mathcal{G}^{\prime}\right) / \operatorname{Lie}\left(\mathcal{G} \times_{R} R^{\prime}\right)\right)
$$

is called the base change conductor of $G$.

Note that our definition differs slightly from the one in [4, 2.4]. Chai extends the tuple of elementary divisors by adding zeroes to the left until the length of the tuple equals the dimension of $G$.

We have $c(G)=0$ iff $G$ has good reduction. If $G$ is tamely ramified, then the elementary divisors of $G$ coincide with the non-zero jumps of Edixhoven's filtration for semi-abelian varieties [6, 4.18]. This comparison result shows in particular that 
$0<c_{i}<1$ for all $i$. We'll now see that the functions $\operatorname{ord}_{G}$ and $u_{G}$ (and hence $t_{G}=g-u_{G}$ ) can be computed from the elementary divisors of $G$. For every real number $x$, we denote by $\lfloor x\rfloor$ the unique integer in the interval $] x-1, x]$.

Proposition 4.7. Assume that $G$ is tamely ramified. Denote by $\left(c_{1}, \ldots, c_{v}\right)$ its tuple of elementary divisors, and by $c(G)$ its base change conductor. For any $d \in \mathbb{N}^{\prime}$ we have

$$
\begin{aligned}
\operatorname{ord}_{G}(d) & =\sum_{i=1}^{v}\left\lfloor c_{i} \cdot d\right\rfloor \\
u_{G}(d) & =\left|\left\{i \in\{1, \ldots, v\} \mid d \cdot c_{i} \notin \mathbb{Z}\right\}\right| .
\end{aligned}
$$

In particular, if $e$ is the splitting degree of $G$, then

$$
\operatorname{ord}_{G}\left(d+e^{\prime}\right)=\operatorname{ord}_{G}(d)+c(G) \cdot e^{\prime}
$$

for each $d \in \mathbb{N}^{\prime}$ and each multiple $e^{\prime}$ of e such that $d+e^{\prime} \in \mathbb{N}^{\prime}$.

Proof. This follows from [6, 6.2 and 7.5].

Corollary 4.8. If $G$ is tamely ramified, then the unipotent rank $u(G)$ of $\mathcal{G}_{s}^{o}$ is equal to the number $v$ of elementary divisors of $G$.

\section{Elementary Divisors AND MONODROMY}

Proposition 5.1. Let $G$ be a tamely ramified algebraic $K$-torus and denote by $c_{1} \leq \ldots \leq c_{v}$ its elementary divisors. Denote by e the splitting degree of $G$, and fix a primitive e-th root of unity $\xi$ in an algebraic closure $\mathbb{Q}^{a}$ of $\mathbb{Q}$. The characteristic polynomial $P_{\sigma}(t)$ of $\sigma$ on $H^{1}\left(G \times_{K} K^{t}, \mathbb{Q}_{\ell}\right)$ is given by

$$
P_{\sigma}(t)=(t-1)^{t_{G}(1)} \prod_{i=1}^{v}\left(t-\xi^{e \cdot c_{i}}\right) \in \mathbb{Z}[t] .
$$

If we put, for each integer $d>1$,

$$
\nu_{d}=\left|\left\{i \in\{1, \ldots, v\} \mid \tau\left(c_{i}\right)=d\right\}\right|,
$$

then the Euler number $\varphi(d)$ divides $\nu_{d}$, and

$$
P_{\sigma}(t)=(t-1)^{t_{G}(1)} \prod_{d>1} \Phi_{d}(t)^{\nu_{d} / \varphi(d)} .
$$

Recall that $\tau\left(c_{i}\right)$ denotes the order of $c_{i}$ in $\mathbb{Q} / \mathbb{Z}$.

Proof. The second expression for $P_{\sigma}(t)$ follows immediately from the first. Note that the product over $d>1$ is finite since $\nu_{d}$ vanishes unless $d$ divides $e$.

So let us prove that (5.1) holds. Consider the Néron model $\mathcal{G}(e)$ of $G(e)=G \times_{K}$ $K(e)$, and denote by $\mathfrak{M}^{\prime}$ the maximal ideal of the normalization $R(e)$ of $R$ in $K(e)$. If we let $G(K(e) / K)$ act on $K(e)$ on the left, then any element $\theta$ of $G(K(e) / K)$ acts on the rank one $k$-vector space $\mathfrak{M}^{\prime} /\left(\mathfrak{M}^{\prime}\right)^{2}$ by multiplication with an element $\theta^{\prime}$ of $\mu_{e}(k)$, and the map $\theta \mapsto \theta^{\prime}$ defines an isomorphism $G(K(e) / K) \cong \mu_{e}(k)$. We denote by $\zeta$ the image of $\sigma$ in $G(K(e) / K) \cong \mu_{e}(k)$. Then by [6, 4.8 and 4.17], the characteristic polynomial of the $\zeta$-action on $\operatorname{Lie}\left(\mathcal{G}(e)_{s}\right)$ equals

$$
Q(t)=(t-1)^{t_{G}(1)} \prod_{i=1}^{v}\left(t-\zeta^{e \cdot c_{i}}\right) \in k[t] .
$$


Since $\mathcal{G}(e)$ is a split $R(e)$-torus, the $k$-vector space $\operatorname{Lie}\left(\mathcal{G}(e)_{s}\right)$ is canonically isomorphic to $\operatorname{Hom}_{\mathbb{Z}}(X, k)$, so that $Q(t)$ equals the image of $P_{\sigma}(t)$ under the morphism $\mathbb{Z}[t] \rightarrow k[t]$.

We know that $P_{\sigma}(t)$ is a product of cyclotomic polynomials $\Phi_{d}(t)$ with $d$ prime to $p$. It remains to show that the only such product mapping to $Q(t) \in k[t]$ is

$$
Q^{\prime}(t)=(t-1)^{t_{G}(1)} \prod_{i=1}^{v}\left(t-\xi^{e \cdot c_{i}}\right) \in \mathbb{Z}[t] .
$$

It suffices to prove that for all tuples $\left(m_{1}, \ldots, m_{r}\right)$ and $\left(n_{1}, \ldots, n_{s}\right)$ of elements in $\mathbb{N}^{\prime}$, the function

$$
R(t)=\frac{\prod_{i=1}^{r}\left(t^{m_{i}}-1\right)}{\prod_{j=1}^{s}\left(t^{n_{j}}-1\right)} \in \mathbb{Z}\left[t, \frac{1}{t^{n}-1}\right]_{n \in \mathbb{N}^{\prime}}
$$

maps to one in $k(t)$ iff $R(t)=1$. This is easily seen by looking at the zeroes of $\prod_{i=1}^{r}\left(t^{m_{i}}-1\right) \in k[t]$ and $\prod_{j=1}^{s}\left(t^{n_{j}}-1\right) \in k[t]$ and using the fact that $\mu_{n}(k)$ is a cyclic group of order $n$ for each $n \in \mathbb{N}^{\prime}$.

Corollary 5.2. If $G$ is tamely ramified and $G$ has purely additive reduction, then

$$
\left|\Phi_{G}\right|=\prod_{d>1} \Phi_{d}(1)^{\nu_{d} / \varphi(d)}
$$

Proof. Apply Lemma 3.4 and Proposition 3.5.

Corollary 5.3. If $G$ is tamely ramified, then its elementary divisors are invariant under isogeny.

Corollary 5.4. If $G$ is tamely ramified, then its base change conductor satisfies

$$
c(G)=\frac{u(G)}{2},
$$

where $u(G)$ is the unipotent rank of $\mathcal{G}_{s}^{o}$.

Proof. Let $c=\left(c_{1}, \ldots, c_{v}\right)$ be the tuple of elementary divisors of $G$. The fact that the right hand side of (5.1) belongs to $\mathbb{Z}[t]$ implies that the map $x \mapsto 1-x$ defines a permutation of $c$. Therefore,

$$
c(G):=\sum_{i=1}^{v} c_{i}=v / 2=u(G) / 2,
$$

where the last equality follows from Corollary 4.8

Remark. Corollary 5.4 is a special case of a much deeper result by Chai, Yu and de Shalit 5, 11.3 and 12.1], stating that for any algebraic $K$-torus $G$, the base change conductor $c(G)$ equals half of the Artin conductor of the $I$-action on $V=X \otimes_{\mathbb{Z}} \mathbb{Q}$. If $G$ is tame, then the Artin conductor simply equals the dimension of $V / V^{I}$, and this is precisely $u(G)$ by Proposition 4.4. If $G$ is not tame, it is no longer true that its elementary divisors are invariant under isogeny (see for instance [4, 8.5(b)]).

Corollary 5.5. If $G$ is tamely ramified, then the determinant $D$ of the $\sigma$-action on $H^{1}\left(G \times_{K} K^{t}, \mathbb{Q}_{\ell}\right)$ equals $(-1)^{u(G)}$. Likewise, the determinant of the $\sigma$-action on $X \otimes_{\mathbb{Z}} \mathbb{Q}$ equals $(-1)^{u(G)}$.

Proof. If $\xi$ is any primitive $e$-th root of unity in $\mathbb{Q}^{a}$, then by Proposition 5.1 we know that $D=\xi^{c(G) e}$. Now the result follows from Corollary [5.4. 


\section{THE GLOBAL MONODROMY PROPERTY FOR ALGEBRAIC TORI}

The following result is a global version for algebraic tori of Denef and Loeser's motivic monodromy conjecture. For the notion of a pole of a motivic generating series, we refer to [16, 4.7] (it requires some care since $\mathcal{M}_{k}$ might not be a domain).

Theorem 6.1. Let $G$ be a tamely ramified algebraic $K$-torus of dimension $g$. Denote by e the splitting degree of $G$, and by $u(G)$ the unipotent rank of the identity component of the special fiber of the Néron model of $G$. The motivic zeta function $Z_{G}(T)$ belongs to

$$
\mathcal{M}_{k}\left[T, \frac{1}{1-\mathbb{L}^{e p} T^{c(G) e p}}\right] .
$$

It has degree zero if $p=1$ and has strictly negative degree if $p>1$. Moreover, the order of the unique pole $s=c(G)$ of $Z_{G}\left(\mathbb{L}^{-s}\right)$ equals one. The cyclotomic polynomial $\Phi_{\tau(c(G))}(t)$ equals $t+(-1)^{u(G)+1}$, and it coincides with the characteristic polynomial $P_{\sigma}^{(g)}(t)$ of the action of the monodromy operator $\sigma$ on $H^{g}\left(G \times{ }_{K} K^{t}, \mathbb{Q}_{\ell}\right)$.

Proof. Denote by $J$ the set of integers in $\{1, \ldots, e p\}$ that are prime to $p$. By Propositions 4.2, 4.4 and 4.7 we can write

$$
\begin{aligned}
Z_{G}(T) & =\sum_{i \in J}\left(\phi_{G}(i) \mathbb{L}^{u_{G}(i)+\operatorname{ord}_{G}(i)}(\mathbb{L}-1)^{t_{G}(i)} T^{i} \sum_{q \geq 0} \mathbb{L}^{c(G) e p q} T^{e p q}\right) \\
& =\sum_{i \in J}\left(\phi_{G}(i) \mathbb{L}^{u_{G}(i)+\operatorname{ord}_{G}(i)}(\mathbb{L}-1)^{t_{G}(i)} T^{i}\right) \frac{1}{1-\mathbb{L}^{c(G) e p} T^{e p}} .
\end{aligned}
$$

So we see that $Z_{G}\left(\mathbb{L}^{-s}\right)$ has a unique pole at $s=c(G)$, of order one (to see that the order is one and not zero, specialize the zeta function with respect to the Poincaré polynomial; see [11, §8]). Since the $\sigma$-action on $H^{g}\left(G \times{ }_{K} K^{t}, \mathbb{Q}_{\ell}\right)$ is the determinant of the $\sigma$-action on $H^{1}\left(G \times_{K} K^{t}, \mathbb{Q}_{\ell}\right)$, it follows from Corollary 5.5 that

$$
P_{\sigma}^{(g)}(t)=t+(-1)^{u(G)+1} .
$$

This polynomial is equal to $\Phi_{\tau(c(G))}(t)$ by Corollary 5.4 .

\section{ACKNowledgement}

I am grateful to the referee for carefully reading the paper and for valuable comments.

\section{REFERENCES}

[1] L. Bégueri. Dualité sur un corps local à corps résiduel algébriquement clos. Mém. Soc. Math. Fr., Nouv. Sér. 4, 121 pp., 1980/81. MR615883(82k:12019)

[2] S. Bosch, W. Lütkebohmert, and M. Raynaud. Néron models. Ergebnisse der Mathematik und ihrer Grenzgebiete, volume 21, Springer, 1990. MR1045822(91i:14034)

[3] S. Bosch and K. Schlöter. Néron models in the setting of formal and rigid geometry. Math. Ann., 301(2):339-362, 1995. MR:1314591 (96h:14035)

[4] C.L. Chai. Néron models for semiabelian varieties: congruence and change of base field. Asian J. Math., 4(4):715-736, 2000. MR 1870655 (2002i:14025)

[5] C.-L. Chai and J.-K. Yu. Congruences of Néron models for tori and the Artin conductor (with an appendix by E. de Shalit). Ann. Math. (2), 154:347-382, 2001. MR1865974(2003e:11126)

[6] L. Halvard Halle and J. Nicaise. Motivic zeta functions of abelian varieties, and the monodromy conjecture. Preprint, arXiv:0902.3755v4. 
[7] F. Loeser and J. Sebag. Motivic integration on smooth rigid varieties and invariants of degenerations. Duke Math. J., 119:315-344, 2003. MR1997948 (2004g:14026)

[8] J. S. Milne. Étale Cohomology, volume 33 of Princeton Mathematical Series. Princeton University Press, 1980. MR:559531 (81j:14002)

[9] E. Nart and X. Xarles. Additive reduction of algebraic tori. Arch. Math. (Basel), 57:460-466, 1991. MR1129520(92m:14056)

[10] J. Nicaise. A trace formula for rigid varieties, and motivic Weil generating series for formal schemes. Math. Ann., 343(2):285-349, 2009. MR2461256 (2010b:14043)

[11] J. Nicaise. A trace formula for varieties over a discretely valued field. To appear in $J$. Reine Angew. Math., arxiv:0805.1323v2.

[12] J. Nicaise. Trace formula for component groups of Néron models. Preprint, arXiv:0901.1809.

[13] J. Nicaise and J. Sebag. The motivic Serre invariant, ramification, and the analytic Milnor fiber. Invent. Math., 168(1):133-173, 2007. MR2285749 (2009c:14040)

[14] J. Nicaise and J. Sebag. Motivic Serre invariants and Weil restriction. J. Algebra, 319(4):15851610, 2008. MR2383059 (2009e:14041)

[15] J. Nicaise and J. Sebag. A note on motivic integration in mixed characteristic. Preprint, arXiv:0912.4887.

[16] B. Rodrigues and W. Veys. Poles of zeta functions on normal surfaces. Proc. London Math. Soc., 87(3):164-196, 2003. MR1978573 (2004c:14022)

[17] J.-P. Serre. Corps locaux. Hermann, Paris, 1962. MR0150130 (27:133)

[18] J.-P. Serre. Groupes algébriques et corps de classes. Hermann, Paris, 1959. MR0103191 $(21: 1973)$

[19] X. Xarles. The scheme of connected components of the Néron model of an algebraic torus. J. Reine Angew. Math., 437:167-179, 1993. MR1212256 (94d:14044)

Laboratoire Painlevé, Université Lille 1, CNRS - UMR 8524, Cité Scientifique, 59655 Villeneuve d'Ascq CÉdex, France

Current address: Department of Mathematics, KULeuven, Celestijnenlaan 200B, 3001 Heverlee, Belgium

E-mail address: johannes.nicaise@wis.kuleuven.be 